\title{
Gallium/aluminum nanocomposite material for nonlinear optics and nonlinear plasmonics
}

\author{
A. V. Krasavin, K. F. MacDonald, A. S. Schwanecke, and N. I. Zheludev ${ }^{\text {a) }}$ \\ EPSRC Nanophotonics Portfolio Centre, School of Physics and Astronomy, University of Southampton, \\ Southampton SO17 1BJ, United Kingdom
}

(Received 25 April 2006; accepted 31 May 2006; published online 21 July 2006)

\begin{abstract}
We report on a new type of composite metallic structure for nonlinear optics and nonlinear plasmonics, created by grain boundary penetration of gallium into an aluminum film. These composite films form mirrorlike interfaces with silica and show an exceptionally broadband phase-transition-based nonlinear response to optical excitation. () 2006 American Institute of Physics. [DOI: 10.1063/1.2234276]
\end{abstract}

The properties of the two materials forming a metaldielectric interface determine its reflectivity and its ability to support surface plasmon-polariton (SPP) waves. ${ }^{1}$ The ability to control these properties using an optical, electrical, electron-beam, or plasmonic signal offers numerous opportunities in optical signal processing and "active plasmonics." ${ }^{2}$ In recent years there has been growing interest in the development of new materials with tailored optical characteristics to provide the basis of emerging photonic technologies. Enhanced and/or novel optical properties can be achieved in random and periodic nano- and microstructured media and in a diverse range of composite materials. ${ }^{3-5}$ Here we introduce a self-assembled nanoscale composite for applications in nonlinear optics and active plasmonics. The material is a polycrystalline aluminum film on a silica substrate wherein the grain boundaries between aluminum domains and the interface between the aluminum and the silica are infiltrated with nanolayers of gallium. Within this composite material, a reversible excitation-induced shift in the equilibrium between the solid and liquid phases of gallium ${ }^{6}$ provides a substantial change in its optical properties, while the framework of aluminum domains provides the foundation of a uniformly high-quality interface with silica. We demonstrate that the optical properties of these composite films, which are easy to manufacture, can be substantially changed in a reversible manner by changing their temperature or stimulating them with laser light.

$\mathrm{The} \mathrm{Ga} / \mathrm{Al}$ composite is manufactured by first coating, by evaporation, a fused silica substrate with a polycrystalline aluminum film $\sim 250 \mathrm{~nm}$ thick. Then, under normal atmospheric conditions, with the substrate held at a temperature of $\sim 33{ }^{\circ} \mathrm{C}$ (a few degrees above gallium's $29.8^{\circ} \mathrm{C}$ melting point), a small drop of liquid gallium (nominal purity 99.9999\%) is placed on the exposed surface of the aluminum. Once the oxide layer on aluminum surface beneath the gallium is breached, the gallium begins to spread itself at a rate of approximately $0.5 \mathrm{~mm} / \mathrm{min}$ across the aluminum surface and penetrates the grain structure of the aluminum film through to the silica interface, where we believe that it forms a layer thicker than the optical skin depth of liquid gallium $(\sim 10 \mathrm{~nm})$. The metals have limited mutual solubility ${ }^{7}$ but the $\mathrm{Ga} / \mathrm{Al}$ system is known for the rapidity of its "grain boundary penetration" (GBP) process. ${ }^{8-11}$ The rate at which

${ }^{a)}$ Electronic mail: n.i.zheludev@ soton.ac.uk; url: www.nanophotonics.org.uk the gallium spreads across the exposed aluminum surface and the micron-scale pattern of "arachnoid" lines produced on the exposed metal surface [see inset in Fig. 1(a)] are consistent with previous investigations. ${ }^{9,10}$ An optical inspection of the silica-metal interface under a polarizing microscope (after solidification of the gallium) reveals large $\left(\mathrm{mm}^{2}-\mathrm{cm}^{2}\right)$ unstructured homogeneous areas in which the gallium's crystalline structure is uniformly oriented. The optical quality of the silica-metal interface is very high but the presence of gallium is easily detected because the silver/grey tone of the $\mathrm{Ga} / \mathrm{Al}$-silica interface is slightly darker than that of the Al-silica interface.

To characterize the linear and nonlinear optical properties of the $\mathrm{Ga} / \mathrm{Al}$ nanocomposite, the sample was mounted on a thermostatic plate. First, the near-normal incidence reflectivity of the $\mathrm{Ga} / \mathrm{Al}$-silica interface was measured as a function of temperature at $633 \mathrm{~nm}$ using a He-Ne laser (circularly polarized to minimize the effect of rotations in the crystallographic orientation of the gallium after melting and recrystallization). These reflectivity data are presented in Fig. 1(a) and reveal a hysteresis similar to that associated with the melting and solidification of pure gallium at a silica interface [Fig. 1(b)], with one notable difference: the reflectivity of the $\mathrm{Ga}-\mathrm{Al} /$ silica interface changes continuously with temperature across the entire measured range below the nominal melting and solidification points $\left(T_{m}\right.$ and $T_{s}$, respectively), where the reflectivity changes abruptly. Pure gallium-silica interfaces do show a premelting reflectivity change due to "surface melting" at the interface, ${ }^{12,13}$ but only at temperatures within a few degrees of the metal's melting point; at lower temperatures their reflectivity remains constant. Measurements of Ga/Al-silica interface reflectivity spectra [Fig. $1(c)$ ] performed with a continuum laser source reveal that a significant change in the optical properties of the composite occurs on melting (as it does in pure gallium ${ }^{14,15}$ ) across a very broad range of wavelengths.

To investigate the nonlinear optical properties of the $\mathrm{Ga} / \mathrm{Al}$ nanocomposite, we studied intensity modulations in the reflected $633 \mathrm{~nm}$ probe beam resulting from stimulation of the interface with a pulsed Nd:YAG (yttrium aluminum garnet) laser (6 ns pulses at a wavelength of $1064 \mathrm{~nm}$ with a repetition rate of $5 \mathrm{~Hz}$ ). The Nd:YAG (pump) laser spot was positioned on the interface so as to encompass the smaller $\mathrm{He}-\mathrm{Ne}$ (probe) spot. Using a photodetector and digital oscilloscope with overall bandwidth of $100 \mathrm{MHz}$, the time dy- 

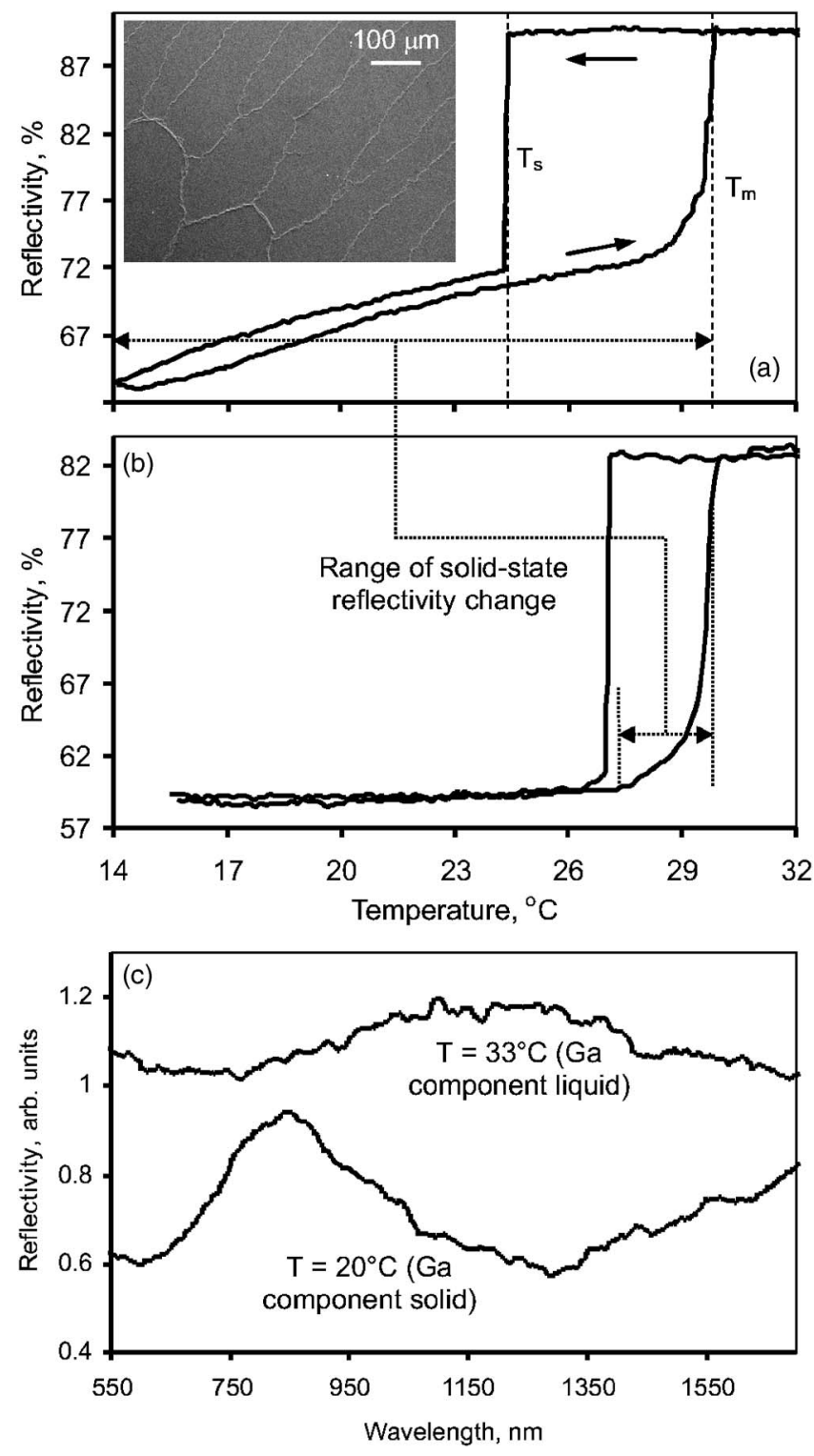

FIG. 1. (a) Temperature dependence of near-normal incidence Ga/Al-silica interface reflectivity at $633 \mathrm{~nm}$. The inset shows a scanning electron microscope image (backscattered electron detection mode) of the "arachnoid" line pattern formed on the surface of the aluminum around the point at which the gallium drop is applied. (b) For comparison, the temperature dependence of near-normal incidence Ga-silica interface reflectivity at $810 \mathrm{~nm}$. (c) Nearnormal incidence reflectivity spectra for a $\mathrm{Ga} / \mathrm{Al}$-silica interface at temperatures of $20^{\circ} \mathrm{C}\left(<T_{s}\right)$ and $33^{\circ} \mathrm{C}\left(>T_{m}\right)$, obtained using a linearly polarized continuum laser source and averaged over a number of points on the $\mathrm{Ga} / \mathrm{Al}$-silica interface.

namics of pump-induced changes in reflectivity were recorded at pump fluences up to $\sim 2 \mathrm{~mJ} / \mathrm{cm}^{2}$ (Fig. 2). On application of a pump pulse there is an immediate increase in the reflected probe intensity. The response time is not resolved in these measurements but we expect the dynamics to be similar to those of pure gallium wherein the initial response occurs within a few picoseconds. ${ }^{16}$ Following termination of a pump pulse, the reflectivity returns to its preexcitation level with a relaxation time ranging from a few tens of nanoseconds to several hundreds of nanoseconds.

The peak magnitude of the induced reflectivity change increases with pump fluence until it reaches a saturation level, as shown in Fig. 3. This behavior may be understood as follows. Through a combination of thermal and nontherDownloaded 25 Jul 2006 to 152.78.192.211. Redistribution subject

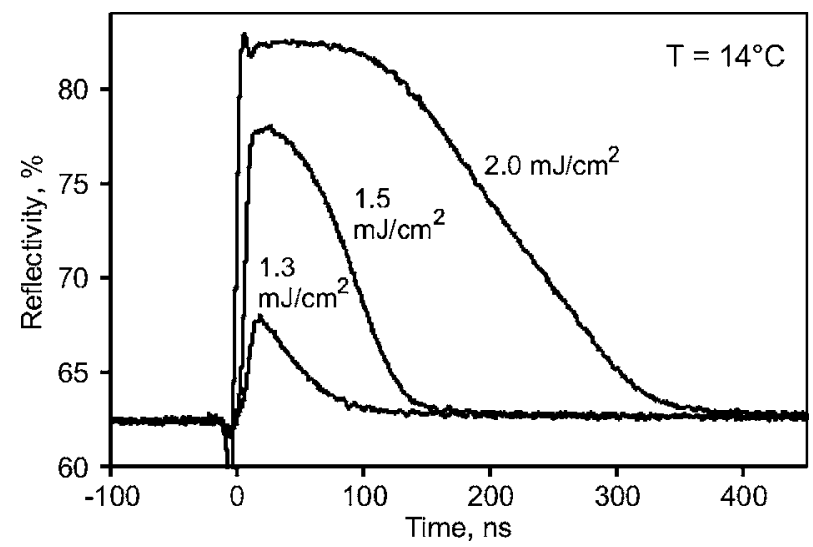

FIG. 2. Dynamics of reflectivity changes at $633 \mathrm{~nm}$ induced in a Ga/Al-silica interface by $6 \mathrm{~ns} \mathrm{Nd}$ :YAG laser pulses at $1064 \mathrm{~nm}$ (labels indicate pump fluence).

mal excitation mechanisms, ${ }^{12,14}$ pump pulses initiate a phase transition from the lower reflectivity solid state ${ }^{17}$ to the higher reflectivity liquid state ${ }^{18}$ in the gallium adjacent to the silica. At higher pump fluences, thicker layers of structurally transformed material are created, giving rise to larger changes in reflectivity. As the thickness of the liquid layer exceeds the skin depth for the probe wavelength, the response saturates. The saturation curve changes with temperature, as shown in Fig. 3, because at temperatures closer to $T_{m}$, less energy is required to induce a transformation in a given volume of material, but the maximum possible reflectivity change is smaller [because the solid-state reflectivity level is higher-see Fig. 1(a)].

The relaxation time of the induced change increases with pump fluence and temperature (see inset to Fig. 3) because at higher fluences and higher temperatures, thicker liquid layers are produced and more time is required for their recrystallization. Moreover, the velocity of the recrystallization front (the boundary between the structurally transformed material and the surrounding solid bulk) decreases as the sample temperature approaches $T_{m}{ }^{19}$

The $\mathrm{Ga} / \mathrm{Al}$ nanocomposite may be used in a range of optical switching applications, ${ }^{15,20,21}$ where it can provide a large area, high-quality silica interface, and good switching contrast. The long-term integrity of the composite remains to

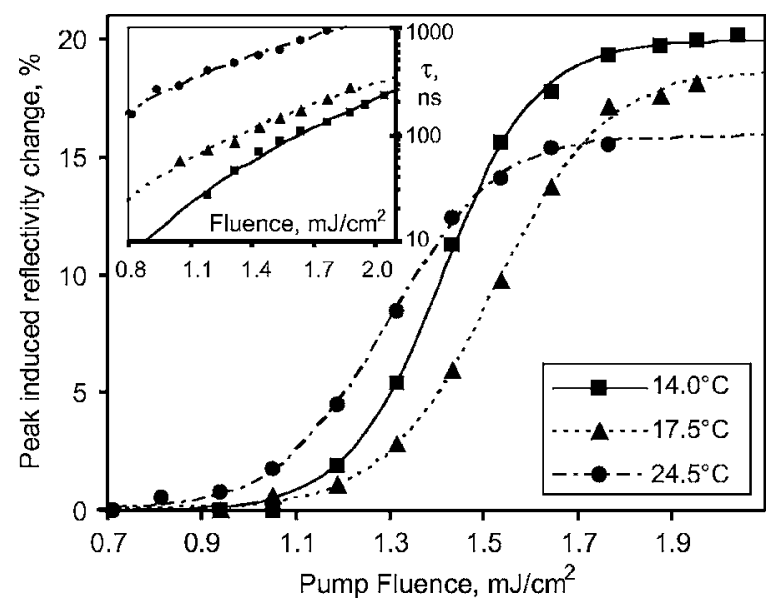

FIG. 3. Peak pump-induced change in absolute reflectivity as a function of pump fluence at three different sample temperatures. The inset shows the corresponding relaxation times as a function of pump fluence.

corresponding relaxation times as a function of pump fluence.
to AlP license or copyright, see http://apl.aip.org/apl/copyright.jsp 
be studied but is likely to depend strongly on the mass ratio of the two metals-an excess of gallium may eventually cause disintegration of the aluminum film. The composite may also find application in plasmonic switching devices such as a recently introduced scheme for active control of SPP wave propagation, ${ }^{2}$ which relies on a reversible change in the dielectric properties of the planar metal-dielectric SPP waveguide. The light-induced structural transition within the gallium component is ideally suited to this application because it is surface driven and provides very good switching contrast: at visible and near-IR wavelengths the SPP decay length on a liquid gallium-silica interface, which ranges from around $5 \mu \mathrm{m}$ at $700 \mathrm{~nm}$ (where it is comparable to the decay length in gold, silver, and aluminum) to about $20 \mu \mathrm{m}$ at $1500 \mathrm{~nm}$, is approximately ten times longer than the decay length on a solid gallium interface.

In conclusion, we have demonstrated that high-quality, optically switchable $\mathrm{Ga} / \mathrm{Al}$-silica interfaces can be formed by penetration of liquid gallium into a thin solid aluminum film on a silica substrate. A reversible light-induced structural transformation in the gallium component of the binary nanolayer can be used to control its optical and electronic properties, and thus to provide a nonlinearity that could be employed in a range of optical and plasmonic switching applications.

${ }^{1}$ W. L. Barnes, A. Dereux, and T. W. Ebbesen, Nature (London) 424, 824 (2003).

${ }^{2}$ A. V. Krasavin and N. I. Zheludev, Appl. Phys. Lett. 84, 1416 (2004).
${ }^{3}$ H. Zhan, C. Zheng, W. Chen, and M. Wang, Chem. Phys. Lett. 411, 373 (2005).

${ }^{4}$ I. Tanahashi, H. Inouye, and A. Mito, J. Ceram. Soc. Jpn. 111, 288 (2003).

${ }^{5}$ W. Wang, G. Yang, Z. Chen, Y. Zhou, H. Lu, and G. Yang, J. Opt. Soc. Am. B 20, 1342 (2003).

${ }^{6}$ P. J. Bennett, S. Dhanjal, P. Petropoulos, D. J. Richardson, N. I. Zheludev, and V. I. Emel'yanov, Appl. Phys. Lett. 73, 1787 (1998).

${ }^{7}$ L. F. Mondolfo, Aluminium Alloys: Structure and Properties (Butterworths, London, 1976).

${ }^{8}$ E. Pereiro-Lopez, W. Ludwig, and D. Bellet, Acta Mater. 52, 321 (2004).

${ }^{9}$ K. Ina and H. Koizumi, Mater. Sci. Eng., A 387-389, 390 (2004).

${ }^{10}$ R. Tanaka, P.-K. Choi, H. Koizumi, and S. Hyodo, Mater. Trans., JIM 42, 138 (2001).

${ }^{11}$ R. C. Hugo and R. G. Hoogland, Scr. Mater. 41, 1341 (1999).

${ }^{12}$ K. F. MacDonald, V. A. Fedotov, R. W. Eason, N. I. Zheludev, A. V. Rode, B. Luther-Davies, and V. I. Emelyanov, J. Opt. Soc. Am. B 18, 331 (2001).

${ }^{13}$ R. Trittibach, C. Grutter, and J. H. Bilgram, Phys. Rev. B 50, 2529 (1994).

${ }^{14}$ K. F. MacDonald, V. A. Fedotov, N. I. Zheludev, B. V. Zhdanov, and R. J. Knize, Appl. Phys. Lett. 79, 2375 (2001).

${ }^{15}$ V. Albanis, S. Dhanjal, N. I. Zheludev, P. Petropoulos, and D. J. Richardson, Opt. Express 5, 157 (1999).

${ }^{16}$ A. V. Rode, M. Samoc, B. Luther-Davies, E. G. Gamaly, K. F. MacDonald, and N. I. Zheludev, Opt. Lett. 26, 441 (2001); 26, 252(E) (2001).

${ }^{17}$ M. Bernasconi, G. L. Chiarotti, and E. Tosatti, Phys. Rev. B 52, 9988 (1995).

${ }^{18}$ N. R. Comins, Philos. Mag. 25, 817 (1972).

${ }^{19}$ S. D. Peteves and R. Abbaschian, Metall. Trans. A 22, 1259 (1991).

${ }^{20}$ P. Petropoulos, H. L. Offerhaus, D. J. Richardson, S. Dhanjal, and N. I. Zheludev, Appl. Phys. Lett. 74, 3619 (1999).

${ }^{21}$ V. A. Fedotov, M. Woodford, I. Jean, and N. I. Zheludev, Appl. Phys. Lett. 80, 1297 (2002). 